\title{
A numerical analysis of heating tissue using the two-temperature model
}

\author{
E. Majchrzak \& Ł. Turchan \\ Institute of Computational Mechanics and Engineering, \\ Silesian University of Technology, Poland
}

\begin{abstract}
The process of heating tissue is considered here. The tissue is treated as a porous medium and is divided into two regions: vascular region (blood vessel) and extravascular region (tissue). The heat conduction in the domain considered is described by the two-temperature model consisting of the system of two coupled equations determining the blood and tissue temperatures. The acceptation of the certain assumptions leads to the model created by the single partial differential equation and the formula concerning the dependence between blood and tissue temperatures. In this equation the coupling factor and phase lag times appear. It should be pointed out that the phase lag times are expressed in terms of the properties of blood and tissue, interphase convective heat transfer coefficient and blood perfusion rate. The equation considered is supplemented by the appropriate boundary and initial conditions. The task has been solved using the finite difference method. In the final part of the paper the results of computations (3D problem) are presented.
\end{abstract}

Keywords: bioheat transfer, two-temperature model, generalized dual-phase lag equation, finite difference method.

\section{Introduction}

Heat transfer in the living biological tissues is associated with the blood perfusion and metabolic heat generation. During the hyperthermia therapy (such a problem is discussed here) the tissue domain is subjected to the external heat source and then the energy equation is supplemented by the component corresponding to the internal heat source associated with the tissue heating. 
The Pennes equation [1] is one of the earliest bioheat transfer equations which describes the temperature distribution in the living tissue

$$
\rho c \frac{\partial T}{\partial t}=\nabla(\lambda \nabla T)+w c_{b}\left(T_{b}-T\right)+Q_{m}+Q_{e x}
$$

where $c$ is the specific heat of tissue, $\rho$ is the density, $\lambda$ is the thermal conductivity, $T$ denotes tissue temperature, $t$ is the time, $w$ is the blood perfusion rate, $c_{b}$ is the specific heat of blood, $T_{b}$ is the arterial blood temperature, $Q_{m}$ is the metabolic heat source and $Q_{e x}$ is the capacity of internal heat sources associated with the external heating of tissue.

The Pennes model assumes that the tissue is supplied with a large number of capillary vessels, the arterial blood temperature $T_{b}$ is uniform throughout the tissue domain and the vein blood temperature is equal to the tissue temperature. In other words, the effect of blood perfusion is assumed to be a homogeneous and isotropic one.

So far, the Pennes equation is widely used, especially in order to predict the temperature distribution during the hyperthermia [2-8] and hypothermia treatment $[2,9-12]$.

It should be pointed out that the Pennes bioheat equation based on the classical Fourier's law of heat conduction which assumes that the thermal disturbance propagates with an infinite speed.

To take into account the heterogeneous structure of biological tissues, the other models of bioheat transfer are developed, for example Cattaneo-Vernotte equation [13, 14] or dual phase lag equation [15-19]. In the Cattaneo-Vernotte model it is assumed that there is a delay between the heat flux vector and the temperature gradient, this means that the temperature gradient always precedes the heat flux vector. In the dual phase lag model (DPLM) either the temperature gradient (cause) precedes the heat flux vector (effect) or the heat flux vector (cause) precedes the temperature gradient (effect) [20]. So, in the first model the relaxation time appears, while in the second model additionally the thermalizaton time occurs. The main problem at the stage of DPLM application is the appropriate estimation of the two phase lag times values [13, 20].

Another group of bioheat transfer models based on the theory of porous media [21, 22]. In this approach the tissue is divided into two regions: the vascular region (blood vessels) and the extravascular region (tissue). Porosity is defined as the ratio of blood volume to the total volume and two equations describing temperature field in the tissue and vessels sub-domains are considered.

Interesting concept is presented in the work [20]. The starting point is the model bases on the theory of porous media. After mathematical manipulations the dual-phase lag bioheat equations with blood or tissue temperature as sole unknown temperature are obtained. It should be pointed out that in this model the phase lag times are expressed in terms of the properties of blood and tissue, interphase convective heat transfer coefficient and blood perfusion rate. This is the reason that the model is called the generalized dual phase lag model. 
In this paper the generalized dual phase lag model is considered. The single partial differential equation describing the tissue temperature and the formula which allows one to determine the blood temperature are taken in to account. The 3D domain of tissue with blood vessels (cube centrally heated) is analysed. The problem is solved using the finite difference method. In the final part of the paper the results of computations are shown and also the conclusions are formulated.

\section{Generalized dual phase lag equation}

The tissue can be treated as a porous medium divided into two regions: the vascular region (blood vessel) and the extravascular region (tissue) [20, 21]. To describe the temperature field in these sub-domains the two-equation porous model $[20,21]$ can be applied

$$
\begin{aligned}
& (1-\varepsilon) \rho_{t} c_{t} \frac{\partial T_{t}}{\partial t}=(1-\varepsilon) \lambda_{t} \nabla^{2} T_{t}+\alpha A\left(T_{b}-T_{t}\right)+ \\
& w c_{b}\left(T_{b}-T_{t}\right)+(1-\varepsilon) Q_{m t}+(1-\varepsilon) Q_{e x}
\end{aligned}
$$

and

$$
\begin{aligned}
& \varepsilon \rho_{b} c_{b}\left[\frac{\partial T_{b}}{\partial t}+\mathbf{u} \cdot \nabla T_{b}\right]=\varepsilon \lambda_{b} \nabla^{2} T_{b}+\alpha A\left(T_{t}-T_{b}\right)+ \\
& w c_{b}\left(T_{t}-T_{b}\right)+\varepsilon Q_{m b}+\varepsilon Q_{e x}
\end{aligned}
$$

where $\varepsilon$ denotes the porosity (the ratio of blood volume to the total volume), $\alpha$ is the heat transfer coefficient, $\mathbf{u}$ is the blood velocity, $A$ is the volumetric transfer area between tissue and blood, $c$ is the specific heat, $\rho$ is the density, $\lambda$ is the thermal conductivity, $T$ denotes temperature, $t$ is the time, $w$ is the blood perfusion rate, $Q_{m}$ is the metabolic heat source and $Q_{e x}$ is the capacity of internal heat sources associated with the external heating of tissue. The subscripts $t$ and $b$ represent the tissue and blood, respectively.

According to the Minkowycz hypothesis [20, 23], before reaching equilibrium, the blood temperature undergoes a transient process described by the relation

$$
\varepsilon \rho_{b} c_{b} \frac{\partial T_{b}}{\partial t}=G\left(T_{t}-T_{b}\right)
$$

where

$$
G=A \alpha+w c_{b}
$$


Parameter $G$ is called the coupling factor. From equation (4) the tissue temperature can be determined

$$
T_{t}=T_{b}+\frac{\varepsilon \rho_{b} c_{b}}{G} \frac{\partial T_{b}}{\partial t}
$$

and

$$
\frac{\partial T_{t}}{\partial t}=\frac{\partial T_{b}}{\partial t}+\frac{\varepsilon \rho_{b} c_{b}}{G} \frac{\partial^{2} T_{b}}{\partial t^{2}}
$$

while

$$
\nabla^{2} T_{t}=\nabla^{2} T_{b}+\frac{\varepsilon \rho_{b} c_{b}}{G} \frac{\partial}{\partial t}\left(\nabla^{2} T_{b}\right)
$$

Adding the equations (2) and (3), the following equation is obtained

$$
\begin{aligned}
& (1-\varepsilon) \rho_{t} c_{t} \frac{\partial T_{t}}{\partial t}+\varepsilon \rho_{b} c_{b} \frac{\partial T_{b}}{\partial t}+\varepsilon \rho_{b} c_{b} \mathbf{u} \cdot \nabla T_{b}=(1-\varepsilon) \lambda_{t} \nabla^{2} T_{t}+ \\
& \varepsilon \lambda_{b} \nabla^{2} T_{b}+(1-\varepsilon) Q_{m t}+\varepsilon Q_{m b}+Q_{e x}
\end{aligned}
$$

Introducing the formulas (6), (7), (8) into equation (9) one has

$$
\begin{aligned}
& C_{e} \frac{\partial T_{b}}{\partial t}+\frac{\varepsilon(1-\varepsilon) \rho_{t} c_{t} \rho_{b} c_{b}}{G} \frac{\partial^{2} T_{b}}{\partial t^{2}}+\varepsilon \rho_{b} c_{b} \mathbf{u} \cdot \nabla T_{b}= \\
& \lambda_{e} \nabla^{2} T_{b}+\frac{\varepsilon(1-\varepsilon) \lambda_{t} \rho_{b} c_{b}}{G} \frac{\partial}{\partial t}\left(\nabla^{2} T_{b}\right)+\varepsilon Q_{m b}+(1-\varepsilon) Q_{m t}+Q_{e x}
\end{aligned}
$$

where

$$
\lambda_{e}=\varepsilon \lambda_{b}+(1-\varepsilon) \lambda_{t}
$$

and

$$
C_{e}=\varepsilon \rho_{b} c_{b}+(1-\varepsilon) \rho_{t} c_{t}
$$

are the effective thermal conductivity and heat capacity, respectively.

The phase lags for heat flux and temperature gradient are defined as [20]

$$
\tau_{q}=\frac{\varepsilon(1-\varepsilon) \rho_{t} c_{t} \rho_{b} c_{b}}{G C_{e}}
$$


and

$$
\tau_{T}=\frac{\varepsilon(1-\varepsilon) \lambda_{t} \rho_{b} c_{b}}{G \lambda_{e}}
$$

Then the equation (10) can be written as follows

$$
\begin{aligned}
& C_{e}\left(\frac{\partial T_{b}}{\partial t}+\tau_{q} \frac{\partial^{2} T_{b}}{\partial t^{2}}\right)+\varepsilon \rho_{b} c_{b} \mathbf{u} \cdot \nabla T_{b}=\lambda_{e} \nabla^{2} T_{b} \\
& +\lambda_{e} \tau_{T} \frac{\partial}{\partial t}\left(\nabla^{2} T_{b}\right)+\varepsilon Q_{m b}+(1-\varepsilon) Q_{m t}+Q_{e x}
\end{aligned}
$$

It should be pointed out that in the dual phase lag equation (15) the blood temperature is unknown and the phase lag times are expressed in terms of the properties of blood and tissue and the coupling factor between blood and tissue. To obtain the bioheat equation where the unknown is only the tissue temperature, the blood temperature is determined from equation (6)

$$
T_{b}=T_{t}-\frac{\varepsilon \rho_{b} c_{b}}{G} \frac{\partial T_{b}}{\partial t}
$$

and this formula is introduced to the equation (15)

$$
\begin{aligned}
& C_{e}\left(\frac{\partial T_{t}}{\partial t}+\tau_{q} \frac{\partial^{2} T_{t}}{\partial t^{2}}\right)+\varepsilon \rho_{b} c_{b} \mathbf{u} \cdot \nabla T_{t}=\lambda_{e} \nabla^{2} T_{t}+\lambda_{e} \tau_{T} \frac{\partial}{\partial t}\left(\nabla^{2} T_{t}\right)+\varepsilon Q_{m b}+ \\
& +(1-\varepsilon) Q_{m t}+Q_{e x}+\frac{\varepsilon \rho_{b} c_{b}}{G}\left[C_{e}\left(\frac{\partial^{2} T_{b}}{\partial t^{2}}+\tau_{q} \frac{\partial^{3} T_{b}}{\partial t^{3}}\right)+\right. \\
& \left.+\varepsilon \rho_{b} c_{b} \mathbf{u} \cdot \frac{\partial}{\partial t}\left(\nabla T_{b}\right)-\lambda_{e} \frac{\partial}{\partial t}\left(\nabla^{2} T_{b}\right)-\lambda_{e} \tau_{T} \frac{\partial^{2}}{\partial t^{2}}\left(\nabla^{2} T_{b}\right)\right]
\end{aligned}
$$

Next, the equation (15) is differentiated with respect to time

$$
\begin{aligned}
& C_{e}\left(\frac{\partial^{2} T_{b}}{\partial t^{2}}+\tau_{q} \frac{\partial^{3} T_{b}}{\partial t^{3}}\right)+\varepsilon \rho_{b} c_{b} \mathbf{u} \cdot \frac{\partial}{\partial t}\left(\nabla T_{b}\right)-\lambda_{e} \frac{\partial}{\partial t}\left(\nabla^{2} T_{b}\right)- \\
& -\lambda_{e} \tau_{T} \frac{\partial^{2}}{\partial t^{2}}\left(\nabla^{2} T_{b}\right)=\varepsilon \frac{\partial Q_{m b}}{\partial t}+(1-\varepsilon) \frac{\partial Q_{m t}}{\partial t}+\frac{\partial Q_{e x}}{\partial t}
\end{aligned}
$$

As it can be seen, the left side of equation (17) is equal to the expression in the brackets on the right side of the equation (18). 
Thus

$$
\begin{aligned}
& C_{e}\left(\frac{\partial T_{t}}{\partial t}+\tau_{q} \frac{\partial^{2} T_{t}}{\partial t^{2}}\right)+\varepsilon \rho_{b} c_{b} \mathbf{u} \cdot \nabla T_{t}=\lambda_{e} \nabla^{2} T_{t}+\lambda_{e} \tau_{T} \frac{\partial}{\partial t}\left(\nabla^{2} T_{t}\right)+ \\
& \varepsilon Q_{m b}+(1-\varepsilon) Q_{m t}+Q_{e x}+\frac{\tau_{q} C_{e}}{(1-\varepsilon) \rho_{t} c_{t}}\left[\varepsilon \frac{\partial Q_{m b}}{\partial t}+(1-\varepsilon) \frac{\partial Q_{m t}}{\partial t}+\frac{\partial Q_{e x}}{\partial t}\right]
\end{aligned}
$$

It can be approximately assumed that [20]

$$
-\varepsilon \rho_{b} c_{b} \mathbf{u} \cdot \nabla T_{t} \approx G\left(T_{b}-T_{t}\right)
$$

and then the generalized dual phase lag equation where the unknown is only the tissue temperature is obtained

$$
\begin{aligned}
& C_{e}\left(\frac{\partial T_{t}}{\partial t}+\tau_{q} \frac{\partial^{2} T_{t}}{\partial t^{2}}\right)=\lambda_{e} \nabla^{2} T_{t}+\lambda_{e} \tau_{T} \frac{\partial}{\partial t}\left(\nabla^{2} T_{t}\right)+G\left(T_{b}-T_{t}\right)+ \\
& \varepsilon Q_{m b}+(1-\varepsilon) Q_{m t}+Q_{e x}+\frac{\tau_{q} C_{e}}{(1-\varepsilon) \rho_{t} c_{t}}\left[\varepsilon \frac{\partial Q_{m b}}{\partial t}+(1-\varepsilon) \frac{\partial Q_{m t}}{\partial t}+\frac{\partial Q_{e x}}{\partial t}\right]
\end{aligned}
$$

\section{Formulation of the problem and method of solution}

The 3D domain of tissue with blood vessels as shown in Figure 1 is considered (cube of side $L$ ). Central part of the domain (cube of side $L / 5$ ) is heated for a period of time $t_{e x}$ in such a way that the capacity of the internal heat sources in this sub-domain is the constant value.

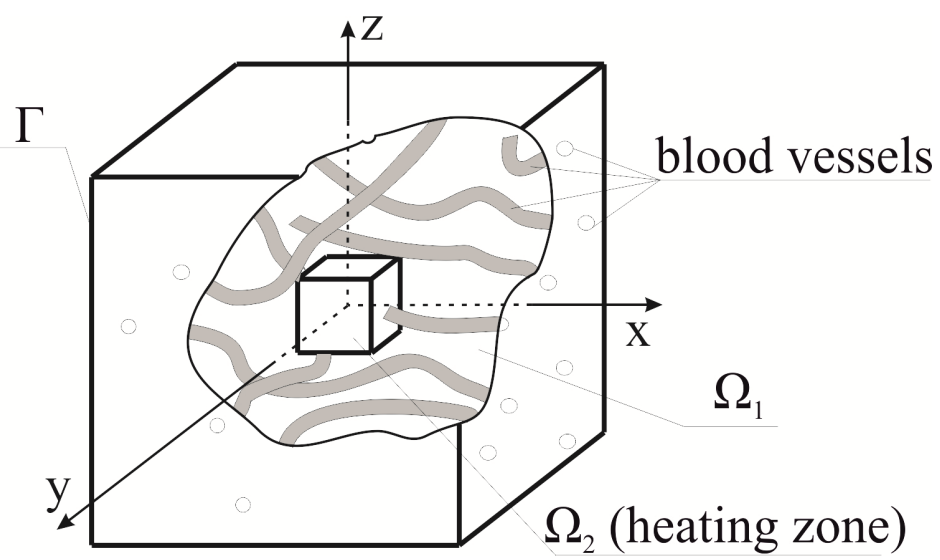

Figure 1: Domain considered. 
Tissue temperature is described by the generalized dual phase lag equation (21), while the blood temperature is determined by the formula (16). On the external boundary of the domain the no-flux condition is taken into account. The initial condition is of the form

$$
t=0: \quad T_{t}=T_{b}=T_{p}, \quad \frac{\partial T_{t}}{\partial t}=0
$$

where $T_{p}$ is the initial temperature of tissue and blood.

The function $Q_{e x}$ in equation (21) connected with the heating is defined as follows (Figure 1)

$$
\begin{cases}(x, y, z) \in \Omega_{1}: & Q_{e x}(x, y, z, t)=0 \\
(x, y, z) \in \Omega_{2}: & Q_{e x}(x, y, z, t)=\left\{\begin{array}{cc}
Q_{0}, & t \leq t_{e x} \\
0, & t>t_{e x}
\end{array}\right.\end{cases}
$$

Assuming the constant values of metabolic heat sources $Q_{m b}, Q_{m t}$ and taking into account the formula (23) the equation (21) can be written in the form

$$
\begin{aligned}
& C_{e}\left(\frac{\partial T_{t}}{\partial t}+\tau_{q} \frac{\partial^{2} T_{t}}{\partial t^{2}}\right)=\lambda_{e} \nabla^{2} T_{t}+\lambda_{e} \tau_{T} \frac{\partial}{\partial t}\left(\nabla^{2} T_{t}\right)+G\left(T_{b}-T_{t}\right)+ \\
& \varepsilon Q_{m b}+(1-\varepsilon) Q_{m t}+Q_{e x}
\end{aligned}
$$

This equation is solved using the explicit scheme of the finite difference method [22]. Let $T^{f}=T_{t}(x, y, z, f \Delta t)$ and $T_{b}{ }^{f}=T_{b}(x, y, z, f \Delta t)$ where $\Delta t$ is the time step. Then, for time $t^{f}=f \Delta t(f \geq 2)$ the following approximate form of equation (24) can be proposed

$$
\begin{aligned}
& C_{e} \frac{T^{f}-T^{f-1}}{\Delta t}+C_{e} \tau_{q} \frac{T^{f}-2 T^{f-1}+T^{f-2}}{(\Delta t)^{2}}=\lambda_{e} \nabla^{2} T^{f-1}+ \\
& \lambda_{e} \tau_{T} \frac{\nabla^{2} T^{f-1}-\nabla^{2} T^{f-2}}{\Delta t}+G\left(T_{b}^{f-1}-T^{f-1}\right)+\varepsilon Q_{m b}+(1-\varepsilon) Q_{m t}+Q_{e x}
\end{aligned}
$$

or

$$
\begin{aligned}
& \frac{C_{e}\left(\Delta t+\tau_{q}\right)}{(\Delta t)^{2}} T^{f}=\frac{C_{e}\left(\Delta t+2 \tau_{q}\right)-G(\Delta t)^{2}}{(\Delta t)^{2}} T^{f-1}-\frac{C_{e} \tau_{q}}{(\Delta t)^{2}} T^{f-2}+ \\
& \frac{\lambda_{e}\left(\Delta t+\tau_{T}\right)}{\Delta t} \nabla^{2} T^{f-1}-\frac{\lambda_{e} \tau_{T}}{\Delta t} \nabla^{2} T^{f-2}+G T_{b}^{f-1}+\varepsilon Q_{m b}+(1-\varepsilon) Q_{m t}+Q_{e x}
\end{aligned}
$$


The uniform grid of dimensions $n \times n \times n$ is introduced and then the finite difference equation for internal node $\left(x_{i}, y_{j}, z_{k}\right)$ has the following form

$$
\begin{aligned}
& \frac{C_{e}\left(\Delta t+\tau_{q}\right)}{(\Delta t)^{2}} T_{i, j, k}^{f}=\frac{C_{e}\left(\Delta t+2 \tau_{q}\right)-G(\Delta t)^{2}}{(\Delta t)^{2}} T_{i, j, k}^{f-1}-\frac{C_{e} \tau_{q}}{(\Delta t)^{2}} T_{i, j, k}^{f-2}+ \\
& \frac{\lambda_{e}\left(\Delta t+\tau_{T}\right)}{\Delta t} \nabla^{2} T_{i, j, k}^{f-1}-\frac{\lambda_{e} \tau_{T}}{\Delta t} \nabla^{2} T_{i, j, k}^{f-2}+G T_{b}^{f-1}+\varepsilon Q_{m b}+(1-\varepsilon) Q_{m t}+Q_{e x}
\end{aligned}
$$

where

$$
\begin{aligned}
& \nabla^{2} T_{i, j, k}^{s}=\frac{T_{i-1, j, k}^{s}-2 T_{i, j, k}^{s}+T_{i+1, j, k}^{s}}{h^{2}}+ \\
& \frac{T_{i, j-1, k}^{s}-2 T_{i, j, k}^{s}+T_{i, j+1, k}^{s}}{h^{2}}+\frac{T_{i, j, k-1}^{s}-2 T_{i, j, k}^{s}+T_{i, j, k+1}^{s}}{h^{2}}
\end{aligned}
$$

while $s=f-1$ or $s=f-2$ and $h$ is the constant grid step.

Finally, the tissue temperature at the node $\left(x_{i}, y_{j}, z_{k}\right)$ is calculated from the formula

$$
\begin{aligned}
& T_{i, j, k}^{f}=\frac{C_{e} h^{2}\left(\Delta t+2 \tau_{q}\right)-G h^{2}(\Delta t)^{2}-6 \lambda_{e} \Delta t\left(\Delta t+\tau_{T}\right)}{C_{e} h^{2}\left(\Delta t+\tau_{q}\right)} T_{i, j, k}^{f-1}+ \\
& \frac{\lambda_{e} \Delta t\left(\Delta t+\tau_{T}\right)}{C_{e} h^{2}\left(\Delta t+\tau_{q}\right)}\left(T_{i-1, j, k}^{f-1}+T_{i+1, j, k}^{f-1}+T_{i, j-1, k}^{f-1}+T_{i, j+1, k}^{f-1}+T_{i, j, k-1}^{f-1}+T_{i, j, k+1}^{f-1}\right)- \\
& \frac{\lambda_{e} \Delta t \tau_{T}}{C_{e} h^{2}\left(\Delta t+\tau_{q}\right)}\left(T_{i-1, j, k}^{f-2}+T_{i+1, j, k}^{f-2}+T_{i, j-1, k}^{f-2}+T_{i, j+1, k}^{f-2}+T_{i, j, k-1}^{f-2}+T_{i, j, k+1}^{f-2}\right)- \\
& \frac{C_{e} h^{2} \tau_{q}-6 \lambda_{e} \Delta t \tau_{T}}{C_{e} h^{2}\left(\Delta t+\tau_{q}\right)} T_{i, j, k}^{f-2}+\frac{(\Delta t)^{2}}{C_{e}\left(\Delta t+\tau_{q}\right)}\left[G T_{b i, j, k}^{f-1}+\varepsilon Q_{m b}+(1-\varepsilon) Q_{m t}+Q_{e x}\right]
\end{aligned}
$$

The stability criterion is as follows

$$
\frac{C_{e} h^{2}\left(\Delta t+2 \tau_{q}\right)-G h^{2}(\Delta t)^{2}-6 \lambda_{e} \Delta t\left(\Delta t+\tau_{T}\right)}{C_{e} h^{2}\left(\Delta t+\tau_{q}\right)} \geq 0
$$

The following approximation of equation (16) is proposed

$$
T_{b i, j, k}^{f-1}=T_{i, j, k}^{f-2}-\frac{\varepsilon \rho_{b} c_{b}}{G} \frac{T_{b i, j, k}^{f-1}-T_{b i, j, k}^{f-2}}{\Delta t}
$$

thus 


$$
T_{b i, j, k}^{f-1}=T_{i, j, k}^{f-2}-\frac{\varepsilon \rho_{b} c_{b}}{G} \frac{T_{b i, j, k}^{f-1}-T_{b i, j, k}^{f-2}}{\Delta t}
$$

The way of boundary condition approximation is discussed, among others, in [24].

Summing up, for each time step the equation (32) describing the change of a blood temperature and the equation (29) describing the tissue temperature should be used.

\section{Results of calculations}

In numerical computations the following values of parameters are assumed: thermal conductivity of blood and tissue $\lambda_{b}=\lambda_{t}=0.5 \mathrm{~W} /(\mathrm{m} \mathrm{K})$, blood density $\rho_{b}=1060 \mathrm{~kg} / \mathrm{m}^{3}$, tissue density $\rho_{t}=1000 \mathrm{~kg} / \mathrm{m}^{3}$, specific heat of blood $c_{b}=3770$ $\mathrm{J} /(\mathrm{kg} \mathrm{K})$, specific heat of tissue $c_{t}=4000 \mathrm{~J} /(\mathrm{kg} \mathrm{K})$, metabolic heat sources $Q_{m b}=Q_{m t}=250 \mathrm{~W} / \mathrm{m}^{3}$, blood temperature $T_{b}=37{ }^{\circ} \mathrm{C}$, initial temperature $T_{p}=37^{\circ} \mathrm{C}$, porosity $\varepsilon=0.0174$ and coupling factor $G=150628.5 \mathrm{~W} /\left(\mathrm{m}^{3} \mathrm{~K}\right)$. The values of phase lag times $\tau_{q}$ and $\tau_{T}$ are determined using the formulas (13) and (14).

The spatial discretization creates $500 \times 500 \times 500$ nodes and time step is equal to $\Delta t=0.01 \mathrm{~s}$. Three heating conditions described in Table 1 have been considered. Figure 2 shows the tissue temperature distribution in the $1 / 8$ of the cube (due to symmetry) after the time 40 seconds and for all variants of the heating.

Table 1: Variants of heating.

\begin{tabular}{|c|c|c|}
\hline Variant & $\begin{array}{c}\text { Power density } \\
Q_{e x}\left[\mathrm{MW} / \mathrm{m}^{3}\right]\end{array}$ & Heating duration $t_{e x}[\mathrm{~s}]$ \\
\hline 1 & 7 & 5 \\
\hline 2 & 3.5 & 10 \\
\hline 3 & 1 & 35 \\
\hline
\end{tabular}

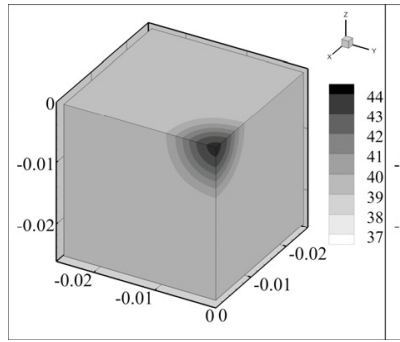

a)

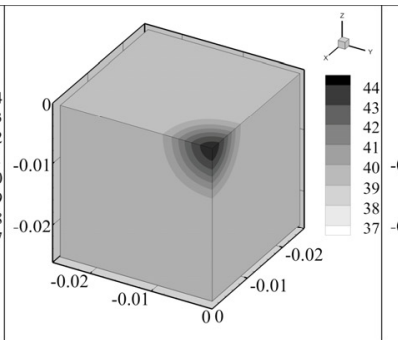

b)

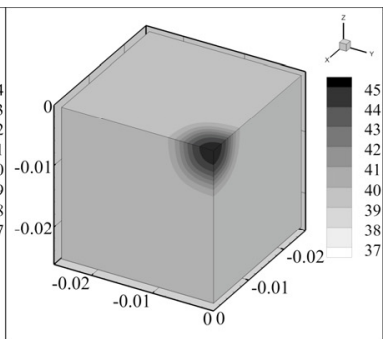

c)

Figure 2: Tissue temperature distribution for time $t=40 \mathrm{~s}(1 / 8$ of the cube): a) 1 st variant, b) 2 nd variant, c) 3 rd variant. 
In Figure 3 the courses of tissue and blood temperatures at the central point of cube for different variants of heating are shown. As can be seen, in each case, the blood temperature and the tissue temperature are similar.

The position of isotherms (tissue temperature) in the central part of crosssection after the time 7 seconds is presented in Figure 4.

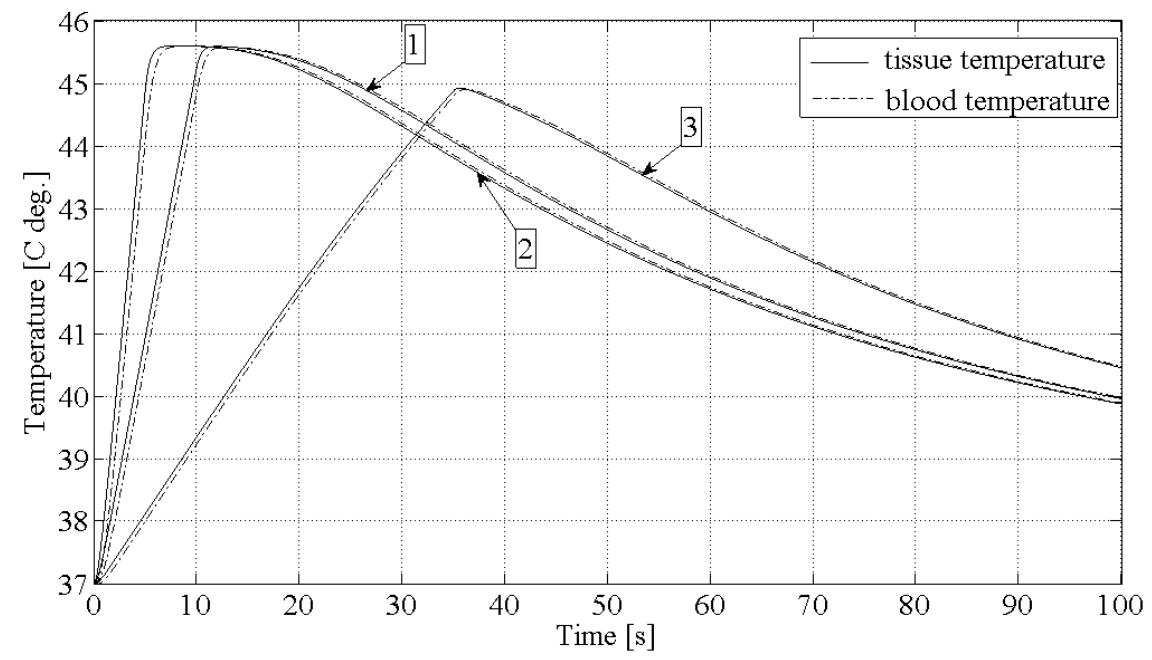

Figure 3: Temperature history at the central point: $1-1$ st variant, $2-2$ nd variant, 3 - 3rd variant.

a)

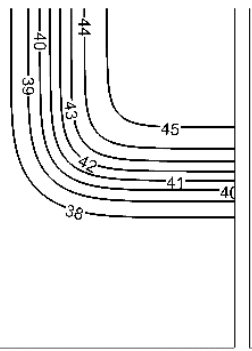

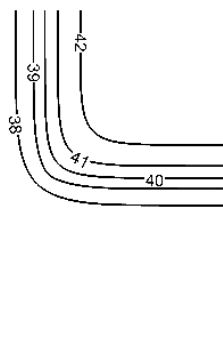

c)

Figure 4: Tissue temperature distribution at the central part of cross section after $7 \mathrm{~s}$, a) 1 st variant, b) 2nd variant, c) 3rd variant.

\section{Conclusions}

To determine the tissue temperature the generalized dual phase lag equation has been used, while the blood temperature has been calculated on the basis of formula describing the relationship between tissue and blood temperatures. 
Occurring in the mathematical model coupling factor and the phase lag times contain the information about the properties of the blood, interphase convective heat transfer coefficient and blood perfusion rate.

The 3D problem concerning the internal heating of the domain considered has been analysed and three variants of heating have been taken into account. At the stage of numerical computations the explicit scheme of a finite difference method has been used. Computations have shown that for the assumed porosity the blood temperature and the tissue temperature differ slightly. In turn, the choice of variant heating has a significant effect on the distributions of temperature and their maximum values in the considered domain.

\section{Acknowledgements}

The paper is a part of the project sponsored by the National Science Centre DEC 2011/01/N/ST6/05231 and project PB3/2013 sponsored by WSZOP.

\section{References}

[1] Pennes H.H., Analysis of tissue and arterial blood temperatures in the resting human forearm, Journal of Applied Physiology, 1, pp. 93-122, 1948.

[2] Majchrzak E., Mochnacki B., Dziewoński M., Jasiński M., Numerical modelling of hyperthermia and hypothermia processes, Advanced Materials Research, 268-270, pp. 257-262, 2011.

[3] Torvi D.A., Dale J.D., A finite element model of skin subjected to a flash fire, Journal of Biomechanical Engineering, 116, pp. 250-255, 1994.

[4] Ng E.Y.K., Chua L.T., Mesh independent prediction of skin burns injury, Journal of Medical Engineering \& Technology, 24, pp. 255-261, 2000.

[5] Jamil M., Ng E.Y.K., Ranking parameters in bioheat transfer using Taguchi parameters, International Journal of Thermal Sciences, 63, pp. 1521, 2013.

[6] Mochnacki, B., Majchrzak E., Sensitivity of the skin tissue on the activity of external heat sources, CMES: Computer Modeling in Engineering and Sciences, 4(3-4), pp. 431-438, 2003.

[7] Mochnacki B., Piasecka Belhayat A., Numerical modeling of skin tissue heating using the interval finite difference method, MCB: Molecular \& Cellular Biomechanics, 10(3), pp. 233-244, 2013.

[8] Jasiński M., Investigation of tissue thermal damage process with application of direct sensitivity method, MCB: Molecular \& Cellular Biomechanics, 10(3), 2013, pp. 183-199, 2013.

[9] Comini G., Giudice L.D., Thermal aspects of cryosurgery, Journal of Heat Transfer, 98, 1976, pp. 543-549, 1976.

[10] Zhao G., Zhang H.F., Guo X.J, Luo D.W., Gao D.Y., Effect of blood flow and metabolism on multidimensional heat transfer during cryosurgery, Medical Engineering \& Physics, 29, pp. 205-215, 2007. 
[11] Yang B., Wan R.G., Muldrew K.B., Donnelly B.J., A finite element model for cryosurgery with coupled phase change and thermal stress aspects, Finite Elements in Analysis and Design, 44, (2008) pp. 288-297, 2008.

[12] Majchrzak E., Dziewoński M., Heat transfer in biological tissue subjected to the action of cylindrical cryoprobe, Moving Boundaries $\mathrm{V}$ : Computational Modelling of Free and Moving Boundary Problems, Book Series: Computational and Experimental Methods, 1, pp. 29-36, 1999.

[13] Kaminski W., Hyperbolic heat conduction equation for materials with a nonhomogeneous inner structure, ASME Journal of Heat Transfer, 112 pp. 555-560, 1990.

[14] Mitra K., Kumar S., Vedavarz A., Moallemi M.K., Experimental evidence of hyperbolic heat conduction in processed meat, ASME Journal of Heat Transfer, 117, pp. 568-573, 1995.

[15] Xu F., Seffen K.A., Lu T.J., Non-Fourier analysis of skin biothermomechanics, International Journal of Heat and Mass Transfer, 51, pp. 2237-2259, 2008.

[16] Majchrzak E., Numerical solution of dual phase lag model of bioheat transfer using the general boundary element method, CMES: Computer Modeling in Engineering and Sciences, 69(1), pp. 43-60, 2010.

[17] Majchrzak E., Application of different variants of the BEM in numerical modeling of bioheat transfer processes, MCB: Molecular \& Cellular Biomechanics, 10(3), pp. 201-232, 2013.

[18] Mochnacki B., Paruch M., Estimation of relaxation and thermalization times in microscale heat transfer model, Journal of Theoretical and Applied Mechanics, 51 (4), pp. 837-845, 2013.

[19] Majchrzak E., Mochnacki B., Greer A.L., Suchy J.S., Numerical modeling of short pulse laser interactions with multi-layered thin metal films, CMES: Computer Modeling in Engineering and Sciences, 41(2), pp. 131146.

[20] Zhang Y., Generalized dual-phase lag bioheat equations based on nonequilibrium heat transfer in living biological tissues, International Journal of Heat and Mass Transfer, 52, pp. 4829-4834, 2009.

[21] Nakayama A., Kuwahara F., A general bioheat transfer model based on the theory of porous media, International Journal of Heat and Mass Transfer, 51, pp. 3190-3199, 2008.

[22] Majchrzak E., Turchan Ł., Numerical analysis of tissue heating using the bioheat transfer porous model, Computer Assisted Methods in Engineering and Science, 20, pp. 123-111, 2013.

[23] Minkowycz, W.J., Haji-Sheikh, A., Vafai, K., On departure from local thermal equilibrium in porous media due to a rapidly changing heat source: the Sparrow number, International Journal of Heat Mass Transfer, 42 (18), pp. 3373-3385, 1999.

[24] Mochnacki B., Majchrzak E., Numerical modeling of casting solidification using generalized finite difference method, Materials Science Forum, 638-642, pp. 2676-2681, 2010. 